\title{
LAS BETAS CALCULADAS, LOS DILEMAS EN SU USO Y EL IMPACTO EN EL CAPM
}

\author{
THE CALCULATED BETAS, THE DILEMMAS IN THEIR USE AND THE IMPACT \\ ON CAPM
}

\author{
Alfredo Aguilar Córdova \\ Magister en Ciencias con mención en Proyectos de Inversión por la Universidad Nacional de Ingeniería - Lima, Perú. \\ Docente de posgrado en la Universidad del Pacífico y en la Universidad de Lima. \\ Email: a.aguilarc@up.edu.pe (Autor Corresponsal) \\ [Recibido: 14/08/2016 Aceptado: 20/11/2016]
}

\begin{abstract}
RESUMEN
La investigación permitió demostrar que las betas calculadas ofrecen diferentes valores dependiendo de la serie histórica de datos seleccionado por el analista, se observan resultados antagónicos con betas del activo más riesgosas que el mercado, pero al ampliar la selección de los datos histórica se convierten en betas menos riesgosas que el mercado en más del $50 \%$ de los casos observados. Los resultados confirman lo señalado por algunos investigadores en el pasado, la beta calculada con datos históricos no es una buena aproximación a la beta de la empresa. Se comprobó el impacto de la beta en el Costo del Capital a través del modelo CAPM en Estados Unidos debido a que existen múltiples betas dependiendo de la serie histórica de datos a tomar. Para tal fin, se ha realizado el cálculo de las betas a través de una regresión lineal utilizando algunas empresas cotizadas en los mercados asiáticos a través de la elección del índice Shanghai Composite, el europeo a través de la elección del índice IBEX 35 y el Norteamericano a través del índice Dow Jones, considerando para todos ellos una frecuencia diaria de los rendimientos y seleccionado una serie histórica de 3 años, 6 meses y 3 meses.
\end{abstract}

\section{PALABRAS CLAVE}

Capital Asset Pricing Model, beta, valorización, riesgo, rendimiento; ética financiera.

\begin{abstract}
The research showed that the calculated betas offer different values depending on the historical series of data selected by the analyst, there are conflicting results with betas of the asset riskier than the market, but when expanding the selection of historical data, betas become less risky than the market in more than $50 \%$ of the observed cases. The results confirm what has been pointed out by some researchers in the past, the beta calculated with historical data is not a good approximation to the Beta of the company. The impact of the beta on the Cost of Capital was verified through the CAPM model in the United States because there are multiple betas depending on the historical series of data to be taken. For this purpose, the calculation of the Betas has been made through a linear regression using some companies quoted in the Asian markets through the choice of the Shanghai Composite index, the European one through the choice of the IBEX 35 and the North American index through the Dow Jones Industrial Average, considering for them a daily frequency of income and selecting a historical series of 3 years, 6 months and 3 months.
\end{abstract}

\section{KEYWORDS}

Capital Asset Pricing Model, beta, valuation, risk, income, financial ethics.

Como Citar: Aguilar, A. (2017). Las betas calculadas, los dilemas en su uso y su impacto en el CAMP. Quipukamayoc, 25(47), 123

- 129. doi: http://dx.doi.org/10.15381/quipu.v25i47.13810 


\section{INTRODUCCIÓN}

El artículo aborda una de las variables, la Beta Calculada, que se necesita para obtener el Costo del Capital $(\mathrm{Ke})$, también denominado Costo del Patrimonio (re) o Rentabilidad Exigida del Accionista (Ke) a través del uso del modelo Capital Asset Pricing Model (CAPM).

A pesar de que muchas variables del Modelo están sujetas a críticas, se ha elegido el Beta debido a su facilidad de cálculo en las empresas cotizadas. La Beta es uno de los elementos controversiales en la aplicación de uno de los métodos más populares para valorizar empresas, el CAPM (Capital Asset Pricing Model) o Modelo de valoración de activos.

"David Levhari y Haim Levy ya señalaron, en un estudio de 1977, que la extensión del intervalo de rendimiento que se considera afecta la medida de beta" (Fornero, 2014, p.120).

Fernández y Carabias (2007) nos revelan en un estudio realizado en España los peligros de utilizar la Beta calculada, se indica que es un error enorme utilizar las betas calculadas con datos históricos para calcular la rentabilidad exigida a las acciones o para medir la gestión de una cartera de valores.

Se pone en tela de juicio a quienes calculan la Beta para aplicarlo al CAPM como paso para descontar los Flujos de Caja de una compañía con fines de valorización, debido a que existe la tentación de utilizar un beta más bajo, luego la tasa de descuento vía el CAPM sería una tasa más baja, así el impacto en la valorización sería más elevada (esto en el caso de que quiera obtenerse el valor más alto para la empresa - posición típica del vendedor), pero también puede ocurrir utilizar un beta más alto, luego la tasa de descuento vía el CAPM sería una tasa más alta, así el impacto en la valorización sería más baja (esto en el caso de que quiera obtenerse el valor más bajo para la empresa - posición típica del comprador). ¿Podría considerarse un problema ético financiero si esperamos que exista un patrón de comportamiento similar por parte de los analistas de diferentes firmas valorizadoras "independientes" que den una recomendación de compra, mantenimiento o venta de acciones sabiendo el dilema de las Betas calculadas?, en caso de ser positiva la respuesta, ¿no debería la entidad reguladora haber tomado cartas en el asunto para evitar que estas firmas valorizadoras den información errática al mercado?.

En el artículo se ha realizado el cálculo de las Betas a través de la regresión lineal utilizando algunas empresas cotizadas en los mercados asiáticos a través de la elección del índice Shanghai Composite, el europeo a través de la elección del índice IBEX 35 y el Norteamericano a través del índice Dow Jones, considerando para todos ellos una frecuencia diaria de los rendimientos y seleccionado una serie histórica de 3 años, 6 meses y 3 meses.

Para una mejor comprensión del tema propuesto, el contenido se ha estructurado en cuatro partes. La primera comprende el marco teórico. En la segunda se plantea la metodología. La tercera abarca los resultados. En la cuarta se aborda la discusión. Luego, se presentan las conclusiones. Finalmente, se da a conocer las Referencias bibliográficas.

Existen muchos métodos para valorizar empresas, uno de los más aceptados en el mercado es el descuento de flujos de caja del accionista que necesita una tasa de descuento apropiada. La fórmula para descontar los flujos de caja del accionista se basa en el Valor Presente:

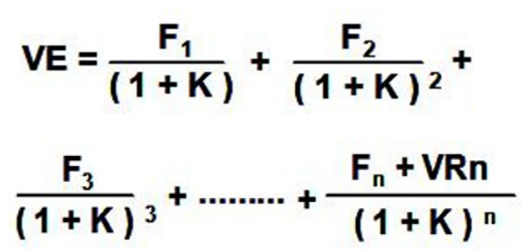

Donde:

VE: Valor del Patrimonio

F1 ... Fn: Flujos de caja del accionista VRN: Valor residual

$\mathrm{K}$ : Tasa de descuento

Como se puede apreciar en la fórmula 1, si la tasa de descuento K aumenta, el Valor del Patrimonio (VE) disminuye, pero si la tasa de descuento $\mathrm{K}$ disminuye, el Valor del Patrimonio (VE) aumenta.

Existen muchas metodologías para determinar la tasa de descuento $(\mathrm{K})$ con el fin de valorizar empresas, la más usada en el mercado es el modelo de valoración de activos financieros, conocido por sus siglas en inglés como Capital Asset Pricing Model (CAPM).

Cruz, Jaulín, y Carmona. (2015) indican que el modelo de valoración de activos financieros (CAPM) es un modelo introducido por Jack L. Treynor, William Sharpe, John Litner y Jan Mossin de forma independiente, basado en trabajos anteriores de Harry Markowitz sobre la diversificación y la Teoría Moderna de Portfolio (p. 177178).

En el CAPM, para trabajar con activos individuales, se hace uso de la recta Security Market Line (SML) la cual representa el rendimiento esperado de todos los activos de un mercado (portafolio de todas las acciones del mercado) como función del riesgo no diversificable y su relación con el rendimiento esperado y el riesgo sistémico (Beta), para mostrar cómo el mercado debe estimar el precio de un 
activo individual en relación con la clase a la que pertenece.

La línea SML permite calcular la proporción del binomio recompensariesgo para cualquier activo en relación con el mercado general.

La relación de equilibrio que describe el CAPM es:

$r_{e}=r_{f}+B\left(r_{m}-r_{f}\right)$

Donde:

$\mathrm{r}_{\mathrm{e}}=$ Retorno requerido del Patrimonio (también conocido como Costo de Capital Ke)

$\mathbf{r}_{\mathrm{f}}=$ Tasa del activo libre de riesgo

$\mathrm{r}_{\mathrm{m}}=$ Retorno del mercado

$\mathrm{B}=$ beta del activo

Para calcular la beta existen varias metodogías que dan el mismo resultado:

$$
\operatorname{Bim}=\frac{\operatorname{Cov}\left(r_{i}, r_{m}\right)}{\operatorname{Var}\left(r_{m}\right)}
$$

Donde:

Bim = Beta del activo (o empresa)

$\operatorname{Cov}\left(\mathbf{r}_{\mathbf{i}}, \mathbf{r}_{\mathbf{m}}\right)$ = Covarianza de los rendimientos del activo (o empresa) contra los rendimientos del mercado (o índice del mercado)

$\operatorname{Var}\left(\mathbf{r}_{\mathbf{m}}\right)=$ Varianza de los rendimientos del mercado (o índice del mercado)

O también se puede usar esta expresión:

\section{$\sigma_{i}$}

$$
\operatorname{Bim}=\rho_{m i}
$$

\section{$\sigma_{m}$}

Donde:

Bim = Beta del activo (o empresa)

$\rho_{\text {mi }}=$ Correlación de los rendimien-

tos del activo (o empresa) contra los rendimientos del mercado (o índice del mercado)

$\sigma_{\mathrm{i}}=$ Desviación típica (o estándar) de los rendimientos del activo (o empresa)

$\sigma_{\mathrm{m}}=$ Desviación típica (o estándar) de los rendimientos del mercado (o índice del mercado)

Como se puede apreciar en la Fórmula 3 , la Beta se puede obtener a través

del cálculo de la Covarianza entre los rendimientos del activo (empresa) y el rendimiento del mercado (índice), la Varianza del mercado (índice), o como se puede apreciar en la Fórmula 4, a través del índice de correlación entre los rendimientos del activo (empresa) y el rendimiento del mercado (índice), la volatilidad del rendimiento del activo (empresa) y la volatilidad del mercado (índice), ambos medidos a través del indicador estadístico desviación típica o estándar.

O también se puede obtener la beta de la empresa a través de una regresión lineal:

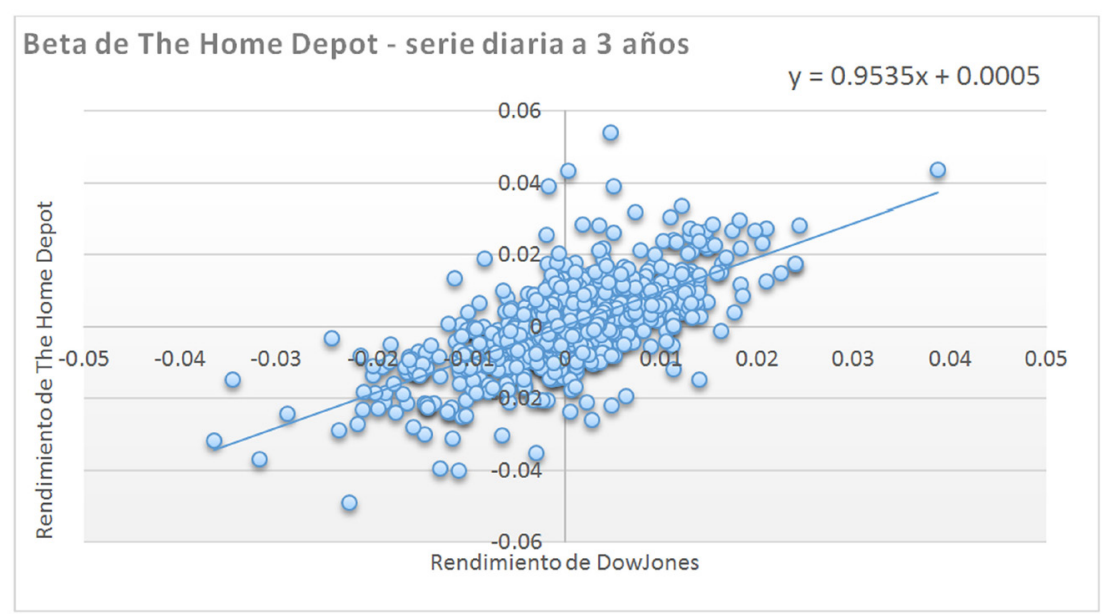

Figura 1. Beta de la empresa The Home Depot

Fuente: Yahoo Finance e Investing / Elaboración: propia

Donde la ecuación: $\mathrm{Y}=0.9535 \mathrm{X}+$ 0.0005 es el resultado de la regresión lineal de la dispersión de puntos combinatorios entre el rendimiento del activo (en este caso la empresa The Home Depot) y el rendimiento del mercado (en este caso el índice Dow Jones). La beta resulta el coeficiente 0.9535 .

Es importante tener presente que se trata de una Beta no apalancada, es decir que se supone que una empresa no tiene deuda en su estructura de capital, por lo tanto, no se incorpora el riesgo financiero, y en caso de querer incorporarlo, debemos determinar una Beta apalancada; así el rendimiento esperado será más alto.

Según Fernández, P (2015), en equilibrio, si todos los inversores tienen idénticas expectativas, todos tendrán la cartera del mercado $\mathrm{M}$, que está en la frontera eficiente (FE). La línea recta RF-M se denomina capital market line (CML):

$E(R i)=R F+[E(R M)-R F] \times[\sigma i / \sigma M]$

Ver Figura 2. 


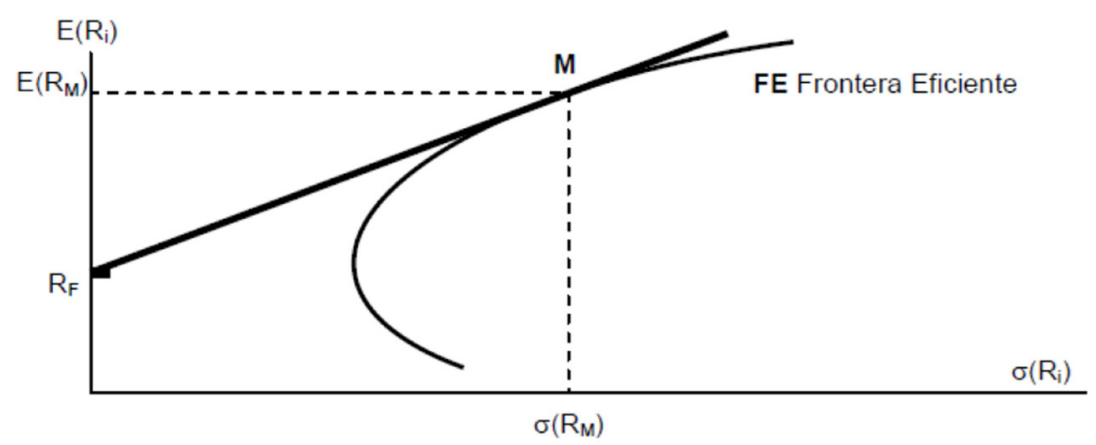

Figura 2. Representación gráfica del CAPM

Fuente: Fernández, P. (2015), CAPM: un modelo absurdo.

Según Sharpe (1962), Bravo (2004) y Fernández (2015), el modelo asume varios aspectos sobre inversores y mercados:

1. Todos los inversores tienen expectativas homogéneas: tienen expectativas homogéneas respecto a las varianzascovarianzas y acerca de los retornos o rendimientos esperados de los activos. Esto quiere decir que todos los inversores tienen las mismas expectativas sobre la rentabilidad futura de todos los activos, sobre la correlación entre las rentabilidades de todos los activos y sobre la volatilidad de todos ellos.

2. Los inversores pueden invertir y tomar prestado a la tasa libre de riesgo (Rf) en cantidades ilimitadas. El mercado de activos es perfecto. No hay asimetrías de información.

3. No hay costes de transacción.

4. Los inversores tienen aversión al riesgo, y maximizan la utilidad de su riqueza en el próximo período.

6. Todos los inversores tienen el mismo horizonte temporal.

7.Los individuos no pueden afectar los precios.

8.El retorno de los activos, se distribuye de manera normal. Explicando el retorno o rendimiento con la esperanza matemática y el riesgo con la desviación estándar.
9.La oferta de activos es fija.

Según Fernández (2015), las consecuencias de usar el CAPM son las siguientes:

1. Cualquier combinación de bonos sin riesgo y de la cartera del mercado domina a cualquier otra combinación de acciones y bonos.

2. Todos los inversores tendrán una cartera compuesta en parte por renta fija sin riesgo y en parte por la cartera del mercado (todos los inversores tendrían la porción de su patrimonio invertida en renta variable muy diversificada). Las proporciones serán distintas según su función de utilidad. 3. La rentabilidad esperada de un activo será igual a la tasa sin riesgo más la beta del activo multiplicada por la rentabilidad exigida al mercado por encima de la rentabilidad de la renta fija sin riesgo. A la expresión [E(RM) - RF] se le denomina prima de riesgo del mercado.

4. La cartera del mercado se compone de todos los activos que existen y la cantidad de cada uno es proporcional a su valor de mercado.

Cabe mencionar que el modelo tradicional del CAPM (con sólo dos términos, ver fórmula 2) no es usado en ningún país de Latinoamérica pues el modelo original está preparado para el mercado norteamericano (Estados Unidos), en consecuencia, al modelo original le agrega otros factores como Prima de riesgo país, Prima de Liquidez, Lambda (factor de ajuste de volatilidades de las bolsas en el país de origen y EE.UU.). Si ya el modelo era considerado "absurdo" (por las hipótesis que no se cumplen) en el mismo mercado norteamericano, peor aún por alterarse con otros factores que fuerzan un uso que no tiene mucho sentido. Según Fernández (2015) la utilización del CAPM provoca innumerables disputas ya que muchas de ellas terminan en juicios y arbitrajes, se aprecian situaciones ridículas con apariencia de científicas tratando de estimar "la verdadera beta" y "la verdadera prima de riesgo" que utiliza "el mercado". Se entiende que son situaciones ridículas porque se trata de buscar dos números que no existen y aunque se calculen deja de tener una aplicación práctica.

Según Mongrut (2007), la aplicación estricta del modelo CAPM (fórmula 2) tiene sentido siempre que el mercado de capitales local o doméstico se encuentre completamente segmentado o aislado de los demás mercados bursátiles en el mundo. Este supuesto ciertamente no se cumple y por lo tanto su aplicación no es conveniente. Independientemente de sus hipótesis o supuestos, que son restrictivos, su aplicación está plagada de problemas debido a que todos sus componentes deben ser estimados de forma prospectiva y la práctica tradicional suele aproximar estas proyecciones a partir de valores históricos. Como complemento restrictivo, la prima histórica por riesgo de mercado en mercados emergentes suele ser negativa producto de la asimetría negativa, exceso de curtosis y excesiva volatilidad de los rendimientos bursátiles. Más aún, pocos títulos en dichos mercados son 
líquidos lo cual impide la estimación adecuada de la beta calculada.

A través de los portales web financieros Yahoo Finance y de Investing se procedió a recolectar la serie histórica diaria de los precios de cinco (5) acciones cotizadas en el mercado europeo (España) a través del IBEX 35 , se recolectaron los precios diarios de cinco (5) acciones cotizadas en el mercado norteamericano (Estados Unidos) a través del Dow Jones y se recolectaron los precios diarios de cinco (5) acciones cotizadas en el mercado asiático (China) a través del Shanghai Composite. Adicionalmente se procedió a recolectar la serie histórica diaria de los índices IBEX35, Dow Jones y Shanghai Composite.

Posteriormente se procedió a calcular los rendimientos utilizando el logaritmo neperiano diario: $\ln (\mathrm{Pt} / \mathrm{Pt}-1)$ de todas las empresas de cada uno de los índices indicados y de los mismos índices.

A estos resultados se le aplicó la Media Aritmética y la Desviación Estándar para cada activo (empresa) y para los índices. Se procedió a calcular el índice de correlación de cada activo (empresa) cotizada con su respectivo índice.

Se aplicó el cálculo de la Beta para cada activo a través de la fórmula 3 y la fórmula 4 indicadas en el apartado anterior, así como la obtención de la ecuación representativa de la dispersión de puntos entre el rendimiento del activo (empresa) y el rendimiento del mercado para obtener la pendiente respectiva (Beta).

En todos los casos se aplicó el análisis a las series diarias a 3 años, 6 meses y 3 meses de cada índice con relación a cada uno de sus 5 activos que operan en dicho mercado.

Tabla 1.

Betas calculadas de cinco activos que conforman el IBEX 35

Fuente: Yahoo Finance. Investing / Elaboración: Propia

\begin{tabular}{cccccc} 
& Iberdrola & Ferrovial & Viscofan & Gamesa & Acciona \\
\hline 3 meses & 0,7805 & 0,9129 & 0,7526 & 0,6554 & 0,7676 \\
\hline 6 meses & 0,6931 & 0,6756 & 0,4348 & 0,7389 & 0,7415 \\
\hline 3 años & 0,6493 & 0,7421 & 0,4316 & 1,1031 & 0,9021 \\
\hline
\end{tabular}

Se puede apreciar que, en algunas acciones del IBEX 35 (Iberdrola, Ferroaños) la Beta disminuye. Sin embargo, sucede lo contrario, vale decir que la Beta aumenta en el caso de Gamesa y Acciona.

histórica diaria del activo aumenta (pasar de 3 meses a 6 meses y hasta 3

\section{Tabla 2.}

Betas calculadas de cinco activos que conforman el Dow Jones

Fuente: Yahoo Finance. Investing / Elaboración: Propia

\begin{tabular}{cccccc} 
& $\begin{array}{c}\text { The Home } \\
\text { Depot }\end{array}$ & Apple & Nike & Pfizer & $\begin{array}{c}\text { Walt } \\
\text { Disney }\end{array}$ \\
\hline 3 meses & 1,2645 & 0,3815 & 0,8650 & 1,4146 & 0,6919 \\
\hline 6 meses & 1,1857 & 0,6612 & 0,9225 & 1,1661 & 0,6799 \\
\hline 3 años & 0,9535 & 1.0085 & 0.9863 & 0,8319 & 1,0016 \\
\hline
\end{tabular}

Se puede apreciar que, en algunas acciones del Dow Jones (The Home Depot y Pfizer), a medida que la data histórica diaria del activo aumenta (pasar de 3 meses a 6 meses y hasta 3 años)

Tabla 3.

Betas calculadas de cinco activos que conforman el Shanghái Composite Fuente: Yahoo Finance. Investing / Elaboración: Propia

\begin{tabular}{cccccc} 
& $\begin{array}{c}\text { Air China } \\
\text { Ltd }\end{array}$ & $\begin{array}{c}\text { Anhui Heli } \\
\text { Co Ltd }\end{array}$ & $\begin{array}{c}\text { Baoding } \\
\text { Tianwei } \\
\text { Baobian } \\
\text { Electric } \\
\text { Co Ltd }\end{array}$ & $\begin{array}{c}\text { Guangdong } \\
\text { Meiyan Jixiang } \\
\text { Hydropower } \\
\text { Co Ltd }\end{array}$ & $\begin{array}{c}\text { Puangzhou } \\
\text { Industrial } \\
\text { Development } \\
\text { Co Ltd }\end{array}$ \\
\hline 3 meses & 0,1119 & 0,0962 & 0,1344 & 0,2201 & 0,0913 \\
\hline 6 meses & 1,2430 & 0,9899 & 1,3621 & 1,4707 & 1,2011 \\
\hline 3 años & 0,9971 & 1,3561 & 1,3343 & 1,3446 & 1,4277 \\
\hline
\end{tabular}

Se puede apreciar que, en todas las acciones del Shanghai Composite, a medida que la data histórica diaria del activo aumenta (pasar de 3 meses a 6 meses y hasta 3 años) la Beta aumenta.

De otro lado, se ha calculado el impacto en el costo del capital (costo de la Beta disminuye. Sin embargo, sucede lo contrario, vale decir que la Beta aumenta en el caso de Apple, Nike y Walt Disney. patrimonio) considerando el cálculo de la beta obtenido para las cinco empresas que conforman el índice Dow Jones a través de la fórmula 2, asumiendo como datos los siguientes:

a)El activo libre de riesgo de Estados Unidos medido a través del Treasury 
Bond a 10 años es 2,49\% (Fuente: Bloomberg)

b)El retorno del mercado es $6.24 \%$

(Damodaran, 2012)

c)Los resultados de los cálculos de las betas de las cinco empresas (activos) que conforman en índice Dow Jones (Ver Tabla 2)

Si aplicamos la tasa de descuento (K) de la Tabla Nro. 4 a la fórmula 1 para valorizar el patrimonio de cualquiera de las cinco empresas, obtendremos diferentes resultados. Mientras más pequeña sea la tasa de descuento $(\mathrm{K})$, más alto será el valor del patrimonio (VE) de las empresas.

\section{CONCLUSIONES}

El modelo CAPM es aplicado ampliamente en el mercado para determinar la tasa de descuento y así valorizar empresas pues se considera una metodología "apropiada" pese a que no se cumplen muchas de sus hipótesis y pese a la inconsistencia de las Betas pues dependen de su serie histórica para su cálculo y también de la frecuencia. Como se puede apreciar en la Tabla Nro 4, la empresa Pfizer es un claro ejemplo real de que no necesariamente una beta de poca data histórica conlleva a una menor tasa de descuento (K).

Por lo tanto, nos enfrentamos a una discusión ético-financiera pues su aplicación no es sólo con fines académicos, sino que se utiliza para hacer una recomendación en el mercado a los inversionistas (comprar, vender o mantener una acción).

También se revela un problema de uso de técnicas en el mercado, pues el CAPM no es la única metodología para calcular la tasa de descuento con fines de valorización.

En el mercado, tanto propietarios de empresas o accionistas mayoritarios, compradores potenciales, analistas de mercado de casas de bolsa y analistas

Tabla 3.

Tasa de descuento (K) obtenida a través del modelo CAPM para diferentes betas calculadas Fuente: Yahoo Finance. Investing / Elaboración: Propia

\begin{tabular}{cccc|c|c} 
& $\begin{array}{c}\text { The Home } \\
\text { Depot }\end{array}$ & Apple & Nike & Pfizer & Walt Disney \\
\hline $\begin{array}{c}\text { Beta a } 3 \\
\text { meses }\end{array}$ & $7,2 \%$ & $3,9 \%$ & $5,7 \%$ & $7,8 \%$ & $5,1 \%$ \\
\hline $\begin{array}{c}\text { Beta a } 6 \\
\text { meses }\end{array}$ & $6,9 \%$ & $5,0 \%$ & $5,9 \%$ & $6,9 \%$ & $5,0 \%$ \\
\hline $\begin{array}{c}\text { Beta a } 3 \\
\text { años }\end{array}$ & $6,1 \%$ & $6,3 \%$ & $6,2 \%$ & $5,6 \%$ & $6,2 \%$ \\
\hline
\end{tabular}

independientes, utilizan discrecionalmente la Beta que más les convenga a sus fines particulares. Por lo tanto, cabe preguntarse si existen conflictos éticos-financieros o es solamente una ignorancia metodológica al no aplicar otros métodos que levanten las restricciones y/o los dilemas intrínsecos en el cálculo de las Betas. Adicionalmente se pone en tela de juicio a quienes calculan la Beta para aplicarlo al CAPM como paso para descontar los Flujos de Caja de una compañía con fines de valorización, debido a que existe la tentación de utilizar una beta más baja, luego la tasa de descuento vía el CAPM sería una tasa más baja, así el impacto en la valorización sería más elevada (esto en el caso de que quiera obtenerse el valor más alto para la empresa - posición típica del vendedor), pero también puede ocurrir utilizar una beta más alta, luego la tasa de descuento vía el CAPM sería una tasa más alta, así el impacto en la valorización sería más baja (esto en el caso de que quiera obtenerse el valor más bajo para la empresa - posición típica del comprador). ¿Podría considerarse un problema ético financiero si esperamos que exista un patrón de comportamiento similar por parte de los analistas de diferentes firmas valorizadoras "independientes" que den una recomendación de compra, mantenimiento o venta de acciones sabiendo el dilema de las Betas calculadas?, en caso de ser positiva la respuesta, ¿no debería la entidad reguladora haber tomado cartas en el asunto para evitar que estas firmas valorizadoras den información errática al mercado?, y desde el punto de vista académico ¿los docentes de finanzas tanto en pregrado como post grado enseñan adecuadamente los pro y contras del modelo CAPM?, ¿los docentes enseñan otras metodologías alternativas para el cálculo de la tasa de descuento apropiada de manera que se vea una gama de resultados con enfoques diferentes y pueda ser aplicado adecuadamente en un análisis de sensibilidad?.

Los resultados del presente artículo, comprueban lo analizado por algunos analistas y académicos del mercado de valores:

La beta calculada con datos históricos no es una buena aproximación a la Beta de la empresa, de esta manera, queda en cuestión la medición del riesgo de la empresa a través de este indicador.

Se cuestiona en el presente artículo sólo uno de los componentes del CAPM (la Beta), por lo que se entiende que existen más factores que afectan a la rentabilidad exigida o costo de patrimonio (re) de una empresa, además de la correlación de los rendimientos de una empresa con los rendimientos del mercado, las volatilidades históricas, la tasa libre de riesgo y 
la prima de riesgo del mercado.

No existe una consistencia en los Betas históricos calculados pues a media que aumenta el periodo las betas se obtienen resultados diferentes, pero aun así se sigue utilizando en el mercado por parte de los "expertos" valorizadores de empresas.

El cálculo del Valor del Patrimonio (Valor del Capital) se ve afectado por el valor de la tasa de descuento, aplicarlo con el CAPM implica tener una inconsistencia porque se obtienen diferentes betas.

No necesariamente una beta calculada con serie histórica corta implica una tasa de descuento (a través del CAPM) más pequeña.

No existe un consenso de mercado para utilizar una serie larga idéntica (por ejemplo 10 años o 5 años o 3 años o menos) porque el modelo original CAPM no lo propone y queda al libre albedrío su elección.

\section{REFERENCIAS BIBLIOGRÁFICAS}

Bloomberg LP. Servicio de software financiero.

Bravo, S. (2004). EL CAPITAL ASSET PRICING MODEL - CAPM: Historia y Fundamentos.[PDF]. Obtenido de http://www.sergiobravo.com/ uploads/publicaciones/files/9.pdf

Damodaran, A. (2012). The Dark Side of Valuation. Presentación de diapositivas. Obtenido de http://people.stern. nyu.edu/adamodar/pdfiles/country/ darkside.pdf

Fernández, P. (2015). CAMP: Un Modelo Absurdo y soluciones alternativas. Trabajo presentado en el International Finance Conference organizada por la American Academy of Financial Management, Bogotá, Colombia.

Fernandez, P. \& Carabias, J. M. (2007). El peligro de utilizar las Betas Calculadas. Documento de Investigación. Obtenido de https://core.ac.uk/download/pdf/6536319.pdf
Fornero, R. (2014). CAPM Cincuenta años de una aventura intelectual. Trabajo presentado en el XXXIV Jornadas Nacionales de Administración Financiera de Universidad Nacional de Cuyo, Mendoza, Argentina.

Mongrut, M. (2007). Valoración de proyectos de inversión en economías emergentes latinoamericanas: el caso de los inversionistas no diversificados. (tesis doctoral). Universidad de Barcelona, Barcelona, España. Recuperado de http://diposit.ub.edu/dspace/ handle/2445/42127

Sharpe, W. (1962). Capital Asset Prices: A Theory of Market Equilibrium under Conditions of Risk. The Journal of Finance, 19(3), pp. 425-442.

Cruz, A., Jaulín, G. y Carmona, D. (2015). Valoración de activos de renta variable para el mercado accionario colombiano en los sectores industrial, comercial y de servicios 2009-2013: modelos fama \& French y Reward Beta. Sinapsis (7), pp. 174-201. 